\title{
DESIGN AND DEVELOPMENT OF A SIMULATOR FOR THE BRAZILIAN DATA COLLECTING SYSTEM BASED ON SATELLITES
}

\author{
Germano Kienbaum \\ Felipe Miranda \\ Associated Laboratory for Computing and Applied Mathematics \\ National Space Research Institute \\ Av. dos Astronautas, 1758 - Jardim da Granja \\ 12227-910, Sao José dos Campos, BRAZIL
}

Hélcio Vieira Junior

Technological Institute of Aeronautics

General-Command for Aerospace Technology

Praça Marechal Eduardo Gomes, 50

12228-900, Sao José dos Campos, BRAZIL

\author{
Joaquim Barreto \\ Marcus Cisotto \\ Wanderson Almeida
}

\author{
Ground Segment Division \\ National Space Research Institute \\ Av. dos Astronautas, 1758 - Jardim da Granja \\ 12227-910, Sao José dos Campos, BRAZIL
}

\begin{abstract}
This work describes an ongoing project, aiming at the development of a complete simulation study of the Brazilian Data Collecting System based on Satellites (SBCD). The SBCD system comprehends a spatial and a ground segment and it was built for automatic acquisition of data of meteorological and hydrological nature for use in environmental and business applications. The main objective of the project is to build a simulator for conducting a thorough analysis of the system, in order to assess its performance, to determine its best configuration and boundary conditions, and to optimize its operational cost and efficiency, in regard to different configurations and operating scenarios.
\end{abstract}

\section{INTRODUCTION}

The modeling and simulation of data communication network systems constitutes a powerful tool, since it provides a way to forecast its behavior before building it and/or to assess its performance without disturbing the operation of the existing system. These techniques are used to study alternative system configurations, making use of analysis designated by "what would happen if ...”, which contribute to improve system components and the networks overall reliability.

The data collecting system used in environmental applications of the MECB (Missão Espacial Completa Brasileira Brazilian Complete Space Mission) is modeled in this work as a data communication network comprehending a set of satellite based nodes, used only for data retransmission, and a set of ground stationary nodes, representing the data collecting platforms, the ground stations and the mission control center, with all nodes being connected by data communication links.

The data is captured by a great number of data collecting platforms spread all over the Brazilian territory and neighboring South American countries, encapsulated in messages sent to and retransmitted by a set of orbiting satellites to a network of ground stations and the mission control center, where it is processed and distributed to the final users, made up of a great number of governmental institutions and enterprises of the business sector.

The questions related with the determination of the number of the data collecting platforms, their distribution on the ground and their satisfactory operation, due to their dependence on the number of satellites and their orbital trajectory, are of a complex and costly nature, and they frequently involve several external partners of the institution, interested in the data acquisition and processing for applications of governmental and business kinds.

The use of simulation techniques and tools turned out to be a good alternative for conducting a thorough analysis of the system, due to its complexity and high setup costs, aiming at designing its optimal parameters and desired performance and the assessment of its alternative configurations and of its operational cost. 


\section{Kienbaum, Miranda, Vieira Junior, Barreto, Cisotto and Almeida}

This work presents the actual development stage of the SBCD simulator prototype and describes the guidelines for project further development, addressing additionally the questions related to the need for changing the prototype original programming language from MODSIM III to SIMSCRIPT III (CACI Products Company 2008), due to the discontinuation of the support to MODSIM III by the software manufacturer.

As an alternative to MODSIM III, CACI has issued a new release of its traditional simulation language SIMSCRIPT, which was renamed SIMSCRIPT III, with facilities for model translation between these languages, powerful new resources for the development of graphical user simulation interfaces and other advanced features based on java programming (CACI Products Company 2008). This new development phase of the ongoing project will also carry out a thorough revision of the simulator prototype and the inclusion of new features.

\section{SBCD SPECIFICATIONS}

The SBCD system allows data acquisition of environmental nature, made by a great number of PCDs (Plataformas de Coleta de Dados - Data Collecting Platforms) spread all over the Brazilian territory.

Beside the PCDs, the system is constituted by a number of ground stations, designated simply as ET (Estações Terrestres - Ground Stations) or ERCDs (Estações de Recebimento e Coleta de Dados - Receiving and Collecting Ground Stations), and the CCM (Centro de Controle de Missão - Mission Control Center), which complement the system ground segment, and by the transponders on board of the satellites, corresponding to its spatial segment. A complete description of the system can be found in Tude, Miranda, Parada, Costa, Pereira, and Medeiros (1986) and Yamaguti, Ribeiro, Becceneri, and Itami (1994). A draft of the system is shown in Figure 1 and it is described briefly in this section.

\subsection{System Generalities}

The system is designed to be able to support up to 2000 PCDs (desirable), but this number and other PCDs configuration's boundary conditions require confirmation, to be initially obtained from the results of the simulation study and later validated by the operation of the real system. The system is also designed to support up to 20 satellites and 20 ground stations. The PCDs may be placed anywhere over the Brazilian territory and their signals need to be received and processed with at least a probability of $95 \%$ success rate during all satellites favorable passages, defined according to clouds cover and other operating conditions.

The favorable satellites passages are defined as these during which the PCDs can make at least three signal transmissions within the mutual visibility period of the three communicating elements PCD - Satellite - Ground Station. The number of favorable passages in 24 hours period depends on the PCD location.

The rate of repetition and power of the message signals sent by the PCDs can be modified and a minimum requirement for installation and operation of a PCD is that it can send at least one message free of errors during its daily operation.

\subsection{The PCDs}

The aim of the PCD is to automatically acquire the data specified for the monitoring of the environment, such as the control of river water flows for issuing early flooding alarms or for irrigation purposes, water quality assessment, management of hydraulic power generation resources, air quality and climate conditions, animal migration, and the use of soil for agricultural purposes.

The data acquisition is made by sensors created according to the specific kind of monitoring desired. The most common equipments are used for measuring quality and level of rivers and water reservoirs, solar radiation, soil temperature and humidity, rainfall and ozone concentration levels, among others.

The data collected, including the PCD identification and actual status, are stored using a standard format and sent to the satellites in orbit. Although this transmission is repeated in certain time intervals, the effective communication only happens when the satellite is over a region in which the simultaneous visibility of all three components is established, namely the PCD, the satellite and the receiving ground station. The PCD signal power lies in a range of 1 to 3 Watts and has a repetition rate of 1 transmission at each 30 to 200 seconds time interval. The energy power supply for the PCD operation is provided by batteries and solar panels.

The PCDs used in the SBCD system can operate in two different message transmission channels, on the frequencies of 401.62 and $401.65 \mathrm{MHz}$. Due to the trajectories of the satellites in orbit, the communication frequencies undergo a deviation of approximately $12 \mathrm{kHz}$ (Doppler's effect). This frequency deviation depends on the PCD location and on the position and velocity of the orbiting satellite at the moment the communication takes place. 


\section{Kienbaum, Miranda, Vieira Junior, Barreto, Cisotto and Almeida}

Due to the randomness of message generation process and the frequency deviations of the signal, the communication channels of the transponders on board of the satellites is considered to be shared by all PCDs that are placed within the simultaneous visibility region of a triple made up of a PCD, a satellite and a ground station in a certain period of time. Therefore, if two or more messages coincide, both in frequency and time of arrival, there will be a collision and all messages are considered lost.

\subsection{The Satellites}

There is no data processing on board of the satellites and there is no capability for storing the data for later transmission in case of real time transmission failure. The transponders on board of the old, but still operating satellites, named SCD1 and SCD2, can only modulate the messages received in the frequencies of $401.65 \mathrm{MHz}$ and $401.62 \mathrm{MHz}$, produce a gain in the signal power, and resend the messages to the CCM (mission control center), located in Cachoeira Paulista, to the ET (ground stations) located in the cities of Cuiabá and Alcântara, using the S band frequency of $2267 \mathrm{MHz}$, and to the ERCD (Receiving and Collecting Ground Stations), distributed over the Brazilian territory and operating on the frequencies of 2267 $\mathrm{MHz}$ and the UHF band of $462.5 \mathrm{MHz}$.

In the case of the satellites CBERS1 and CBERS2 (already in operation) and other future satellites, like the planned SCD3, the messages received from the PCDs will also be retransmitted directly to the ERCDs placed within the region of simultaneous visibility for the triple PCD-satellite-ERCD, and the transmissions will operate both on the S band frequency of $2267 \mathrm{MHz}$, as well as on the UHF band of $462.5 \mathrm{MHz}$.

The ground stations operating on the S band alone (CCM and ETs) and those operating both on the S and UHF bands (ERCDs) receive the signals and process them for detection and identification of the PCDs. When a PCD signal is detected, a channel is allocated for demodulation and processing of the message by the ground station, for them to be used locally (ERCDs) or retransmitted to the mission control center (CCM) in Cachoeira Paulista, for treatment, storage in databases and later distribution to the final users.

The data is made available for the final users both in its original format (directly on the ERCDs), as well as via the Internet, after filtering and unity transformation, realized by the CCM in Cachoeira Paulista. The data must be available for final user within an interval of 30 (thirty) minutes after the satellite passage over the region of interest.

\subsection{The Ground Stations}

There are two types of receiving ground stations, the first one operating on the $\mathrm{S}$ band on $2267 \mathrm{MHz}$, simply denominated ET (ground stations) located in the cities of Cuiabá and Alcântara and the second one, which can both operate on the S band, on $2267 \mathrm{MHz}$, or in the UHF band, on $462.5 \mathrm{MHz}$, denominated ERCD (receiving and collecting ground stations).

The two types of ground stations available correspond to different kinds and cost of the technology used, the ET type that operates on the S band making use of a stationary, more powerful and expensive antenna, and the ERCD type operating on the UHF band making use of a low cost and portable antenna. The receiving ground stations of both kinds can receive and process the signals from the data collecting platforms as well as the signal from other ground stations and satellites from the network denominated as the international ARGOS system.

The ERCD ground station consists of a PC microcomputer autonomously adapted by the user for reception and processing of the data sent by the data collecting platforms, which are retransmitted by the low orbit satellites component of the SBCD and ARGOS systems. The ERCD ground station may process simultaneously the signals of more than one PCD, depending on the number of available channels it has. The PC microcomputer executes all functions dealing with channel designation, configuration and monitoring of the hardware, data bases storage and communication facilities.

The data acquired are stored in databases and made available for the final users in its original format, for direct access from the ET and ERCD, or after they have been formatted (unity transformation) and organized in a way uniquely done by the mission control center. The final users may access these databases respectively via FTP protocol, using a normal PC directly connected to the ERCD, using the public telephone network, or by means of an internet connection established with the mission control center.

\section{SIMULATORS ARCHITECHTURE AND MAIN FUNCTIONAL REQUIREMENTS}

The computational simulation model must represent all processes existing in the SBCD system, from the message generation process by the PCDs, the uplink to the satellite, the frequency transposition and signal power gain on board of the satellites made by the transponders, the downlink to the ground stations in Cuiabá and Alcântara or to the other receiving ground 


\section{Kienbaum, Miranda, Vieira Junior, Barreto, Cisotto and Almeida}

stations ERCDs, and finally the data reception and treatment made by ground stations, especially the mission control Center in Cachoeira Paulista. A complete description of the SBCD simulator's requirements specifications can be found in Itami, Yamaguti, and Kono (1995).

A prototype of the computational simulation model of the SBCD system has already been built making use of the simulation language MODSIM III (CACI Products Company 1997) and it was presented in a previous work (Travassos, Kienbaum, Hirata, and Abdurahiman 2001). MODSIM III is a discrete event simulation language based on the object oriented methodology possessing advanced resources for the development of simulators with graphical user interfaces.

Figure 2 presents a general structure of the simulator main components and their interactions. In the following, a brief analysis of the main components is made to specify their functional requirements and to fix the guidelines for their further development.

The graphical user interface allows the interactive building of the system's configuration scenarios, making use of drag and drop facilities for positioning the elements, dialog boxes for entering its parameters of interest, as well as establishing simulation main execution parameters. It must also provide flexibility for the definition of elements components of virtual missions and to contemplate the case of satellites still to be launched.

The graphical user interface makes use of homogeneous display of graphical windows, data input dialog boxes and command menus for all operations of creation, specification and execution done with the simulator. It is also able to display graphical and textual presentation of the simulation results. Default parameters need to be used to help users in case of incomplete model definition.

Besides the graphical representation of the components of the SBCD system, the user interface must allow the animation of all their static and dynamic entities, such as the PCDs, with their location automatically stated when they are inserted on a map with their appropriate coordinate, represented in some alternative coordinate systems, as well as the generation of the satellites trajectories from a set of parameters characteristic to their insertion in orbit, and the location of the receiving ground stations using the same PCD locating procedure.

The main module must allow the management of all simulator operations, including interrupting the simulation runs and restarting the system from a set of stored values. The main module functionalities must also include basic tasks related with the simulation study, such as project of experiment facilities, and data collection for presentation of results from the simulation runs.

The main module is further responsible for processing and monitoring the performance of important systems values, such as: establishing the communication links from a set of equations which takes into account the common visibility region computed from the movement of the satellites during its passage over this region; the number and the distribution of the valid messages generated by a PCD or set of PCDs received in a certain time period or in a daily operation for a specific ground station, transmitted by a specific satellite or processed and stored by the system as a whole, among other values of interest.

The main module must also provides facilities for error treatment, resulting both from abnormal behavior of the model, as well as from random deviation of elements behavior, such as distortions created by satellite orbit insertion, uncertainties concerning position and speed of satellites, as well as bad alignment of antennas caused by eventual malfunctioning behavior of satellites in operation.

The simulation runs may need to be interrupted for parameters redefinition in an interactive way and the monitoring of the state variables need to be done by means of displays during simulation execution.

The simulation model must allow simultaneous operation of more than one satellite and more than one ground station and the response time for a simulation run may not exceed 5 minutes for each satellite's passage over a network of 400 data collecting platforms.

Finally, the entire simulator needs to be supported by a user's online help.

\section{SIMULATORS ACTUAL DEVELOPMENT STAGE AND DISCUSSIONS}

A prototype of the SBCD Simulator containing these basic functionalities had been already implemented in the simulation language MODSIM III (Travassos 2001). The following modules were used for model structuring and complexity decomposition:

- $\quad$ MSBCD - Main Module of the Brazilian Data Collecting System based on Satellites;

- MAPWIN - Exhibition and Control Module;

- PCD - Data Collecting Platforms Module;

- $\quad$ SATELLITE - Satellite Module; 


\section{Kienbaum, Miranda, Vieira Junior, Barreto, Cisotto and Almeida}

- $\quad$ ERCD - Receiving Ground Stations Module;

- $\quad$ RESULT - Results Presentation Module;

- $\quad$ HELP - Help Module.

The complete description and source code corresponding to these modules written in MODSIM III can be found in Travassos (2001). The code extension of this prototype is about one thousand lines.

The operation of the SBCD simulator prototype is organized according to four main modes, designated as use cases:

- Scenario Building (internally divided into scenario configuration and entity initialization);

- $\quad$ Project of Experiments;

- Simulation (internally divided into execution initialization, simulation, exhibition and control, results generation);

- Analysis and Results Presentation.

The mode Scenario Building deals with the data input for the configuration of the scenario, the graphical elements corresponding to the PCDs, the satellites and the ground stations, with their respective attributes.

The mode Project of Experiments deals with the input of the additional parameters associated with the experimentation to be carried out through the execution of several simulation runs.

The mode Simulation is entered after scenario building and project of experiments are completely specified and it corresponds to the execution and control of all dynamics of the model, the interactive carry out of the simulation run, the monitoring of the active objects and the specification of reports to be generated for analysis of model behavior.

Finally, the mode Analysis and Results Presentation makes use of the database of results generated in the previous phase to display the results organized according to the user predefined types of reports, so that he can reach conclusions about important model parameters and issue recommendations for system improvement and/or new forms of experimentation to deepen the analysis.

The main activity concluded since the project was restarted was the transcription of the model, from MODSIM III to SIMSCRIPT III, a necessary and important step due to the support discontinuity of the manufacturer of MODSIM III and the launching of a new release of SIMSCRIPT III, with powerful resources based on Java programming and facilities for helping the translation between these simulation languages.

The model transcription from MODSIM III to SIMSCRIPT III required two distinct steps, the first one performed by use of an automatic converter developed by the software manufacturer which translates the source code of MODSIM III to SIMSCRIPT III and preserves the same model structure, based on the module decomposition scheme used in MODSIM III, made by separate descriptions of the objects classes definitions and implementations. The second step requires a reorganization of the generated source code into the component structure of the programs directly written in SIMSCRIPT III, in order to clean same overhead resulting from the direct translation and to build a project that better reflects the way SIMSCRIPT III projects are built using the Simstudio graphical interface. Figure 3 shows the main window of the SBCD simulator in this new phase of development after transcription of the code to SIMSCRIPT III.

This transcription phase has been accomplished satisfactorily and a new step has just been started, towards the validation of the features already implemented and the inclusion of new ones, aiming at the complete implementation of all requirements specification for the SBCD simulator described in section 2 of this work.

\section{CONCLUSION}

This work presented an ongoing project, aiming at conducting a complete simulation study of the Brazilian Data Collecting System based on Satellites. The SBCD system consists of a spatial and a ground segment and it was built for automatic acquisition of data of meteorological and hydrological nature for use in environmental and business applications.

A prototype of the computational simulation model of the SBCD system had already been built, making use of the simulation language MODSIM III. Due to the discontinuation of the support to MODSIM III by the software manufacturer, a change of the simulation programming language used for implementation of the original simulator's prototype turned out to be a very important issue for project further development. The model transcription phase has been accomplished successfully and the prototype in SIMSCRIPT III will now be submitted to a review and further development aiming at the full implementation of systems specifications described in this work.

As a last additional advantage of model transcription, one can point out that the computational model in SIMSCRIPT III looks closer to a natural language description of the system, due to SIMSCRIPT III simulation language syntatics and semantics. 
Kienbaum, Miranda, Vieira Junior, Barreto, Cisotto and Almeida

\section{ACKNOWLEDGMENTS}

The authors acknowledge the National Research Council (Conselho Nacional de Pesquisas) for the grant received from the Institutional Qualification Program for Technical Visits and Training Courses of the Brazilian Science and Technology Ministry (MCT) by the first author and the grant received from the Scientific Research Traineeship Program by the second author in support for the development of this research.

\section{REFERENCES}

CACI Products Company 1997. MODSIM III - The language for object oriented programming (users' manual). La Jolla, CA: CACI Products Company.

CACI Products Company 2008. SIMSCRIPT III - User and reference manuals. La Jolla, CA: CACI Products Company. Itami, S. N., W. Yamaguti, and J. Kono. 1995. Data collection system simulator requirements specification. Technical report, INPE - National Space Research Institute .

Travassos, P. R. N. 2001. A simulation study of the brazilian data collecting system based on satellites. Master's thesis, Graduate Program in Applied Computing - INPE - National Space Research Institute .

Travassos, P. R. N., G. S. Kienbaum, C. M. Hirata, and V. A. Abdurahiman. 2001. A simulation study of the brazilian data collecting system based on satellites. In XXXIII Brazilian Operational Research Symposium.

Tude, E. A. P., C. A. I. Miranda, L. E. M. C. Parada, M. H. M. Costa, S. P. Pereira, and V. M. Medeiros. 1986. Analysis of MECB/SS data collecting system. Technical Report INPE-3820-NTE/253, INPE - National Space Research Institute .

Yamaguti, W., E. A. Ribeiro, J. C. Becceneri, and S. N. Itami. 1994. Collection and treatment of the environment data with the brazilian satellite SCD1. Journal of the Brazilian Society of Mechanical Science Special Issue:205-212.

\section{AUTHOR BIOGRAPHIES}

GERMANO KIENBAUM is a senior researcher within the Associated Laboratory for Computing and Applied Mathematics of the National Space Research Institute, Brazil. His Ph.D. is in Computer Science from Brunel University, United Kingdom, in 1995. His research interests include simulation modeling, the development of discrete event simulators and their applications, educational technology and the development of e-learning systems, and business process management. His email is <kienbaumeuol.com.br $>$.

FELIPE MIRANDA is finishing the undergraduation course in Aeronautical Engineering at UNIP (Universidade Paulista). He received a grant from the Scientific Research Traineeship Program, sponsored by the National Research Council, given to undergraduate students for conducting research studies under senior researchers supervision in accredited research institutions and universities. His email is <fel_miranda@hotmail.com>.

HÉLCIO VIEIRA JUNIOR is Bachelor in Aeronautical Science at AFA (Air Force Academy), graduated in 1991. He is MSc in Production Engineering at Federal University of Rio de Janeiro, in 2003. He is a PhD research student at ITA (Instituto Tecnológico de Aeronáutica - Technological Institute of Aeronautics). His email is <junior_hv@yahoo.com.br>.

JOAQUIM BARRETO is Bachelor in Computer Science at UNIVAP (Universidade do Vale do Paraba), graduated in 2001. He belongs to the R\&D team on the SBCD at the DSS (Ground Control Division). His email is is <joaquimedss. inpe.br>.

MARCUS CISOTTO is Bachelor in Eletronical Engineering at UNICAMP (Universidade de Campinas), graduated in 1981. He belongs to the R\&D team on the SBCD at the DSS (Ground Control Division). His email is <marcao@dss. inpe.br>.

WANDERSON ALMEIDA is Bachelor in Computer Science at Faculdades Integradas de Caratinga, MG, graduated in 2007. He belongs to the R\&D team on the SBCD at the DSS (Ground Control Division). His email is <wanderson@dss. inpe.br>. 


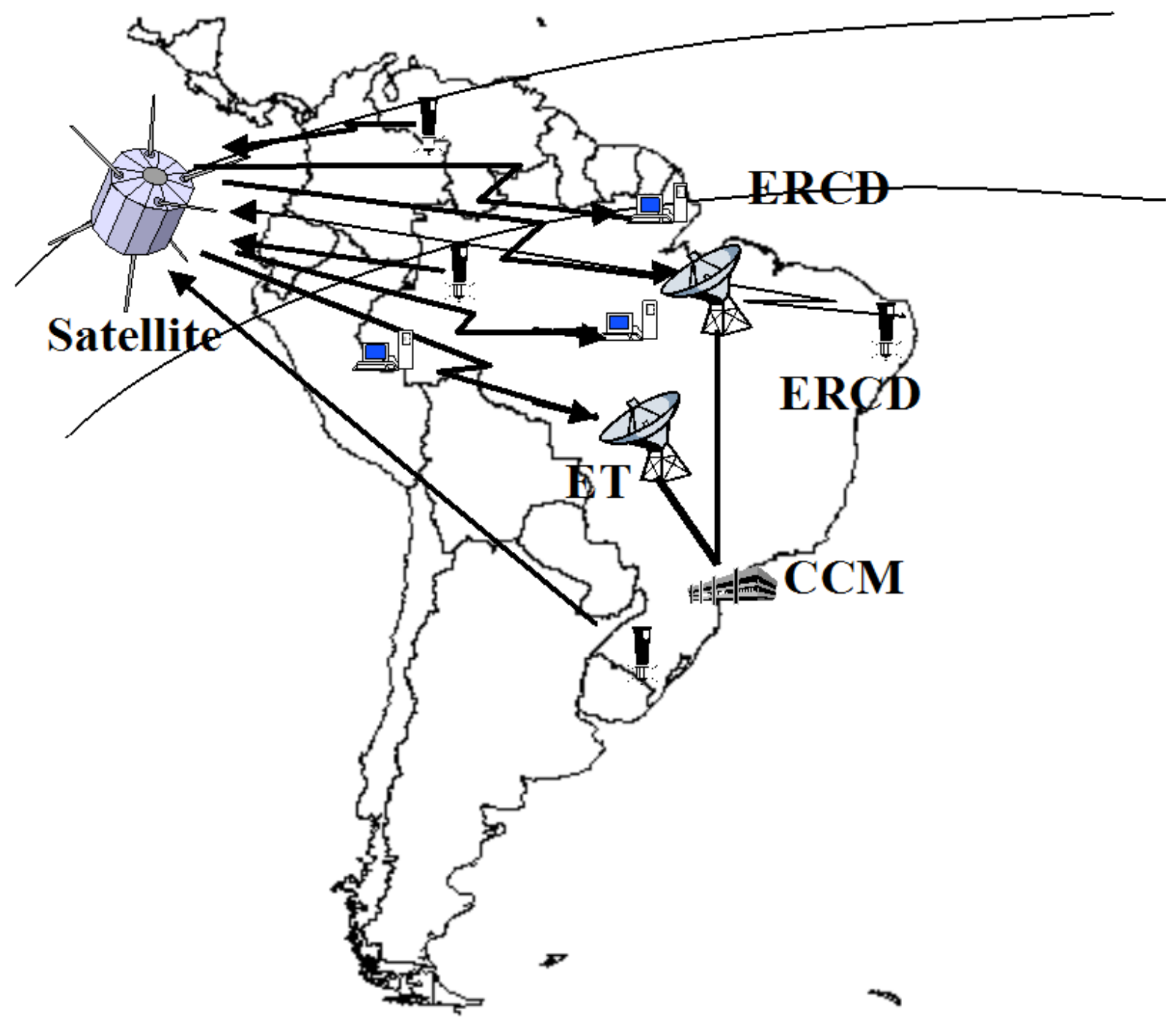

Figure 1: The SBCD General Layout 
Kienbaum, Miranda, Vieira Junior, Barreto, Cisotto and Almeida

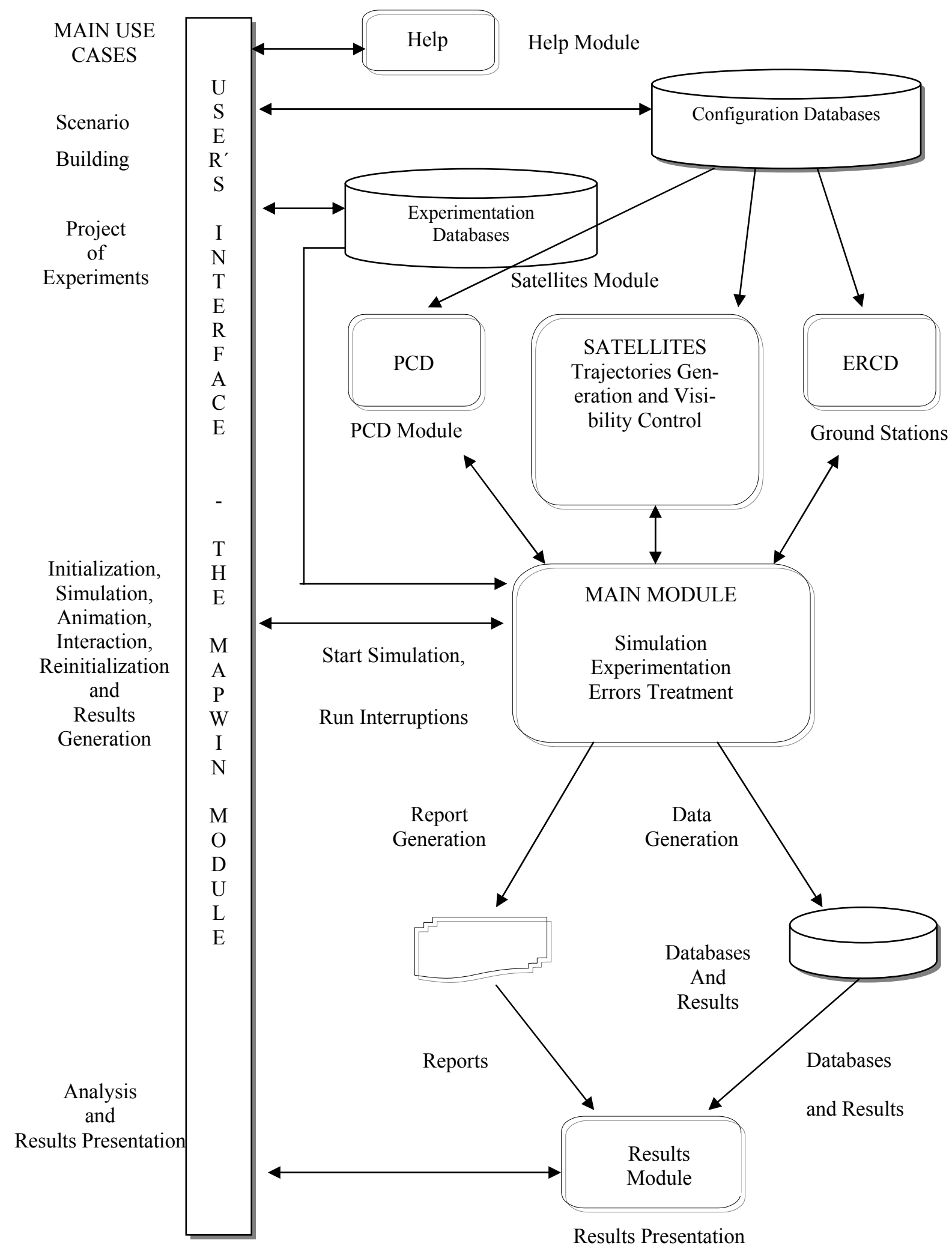

Figure 2: Architecture of the SBCD simulator prototype 
Kienbaum, Miranda, Vieira Junior, Barreto, Cisotto and Almeida

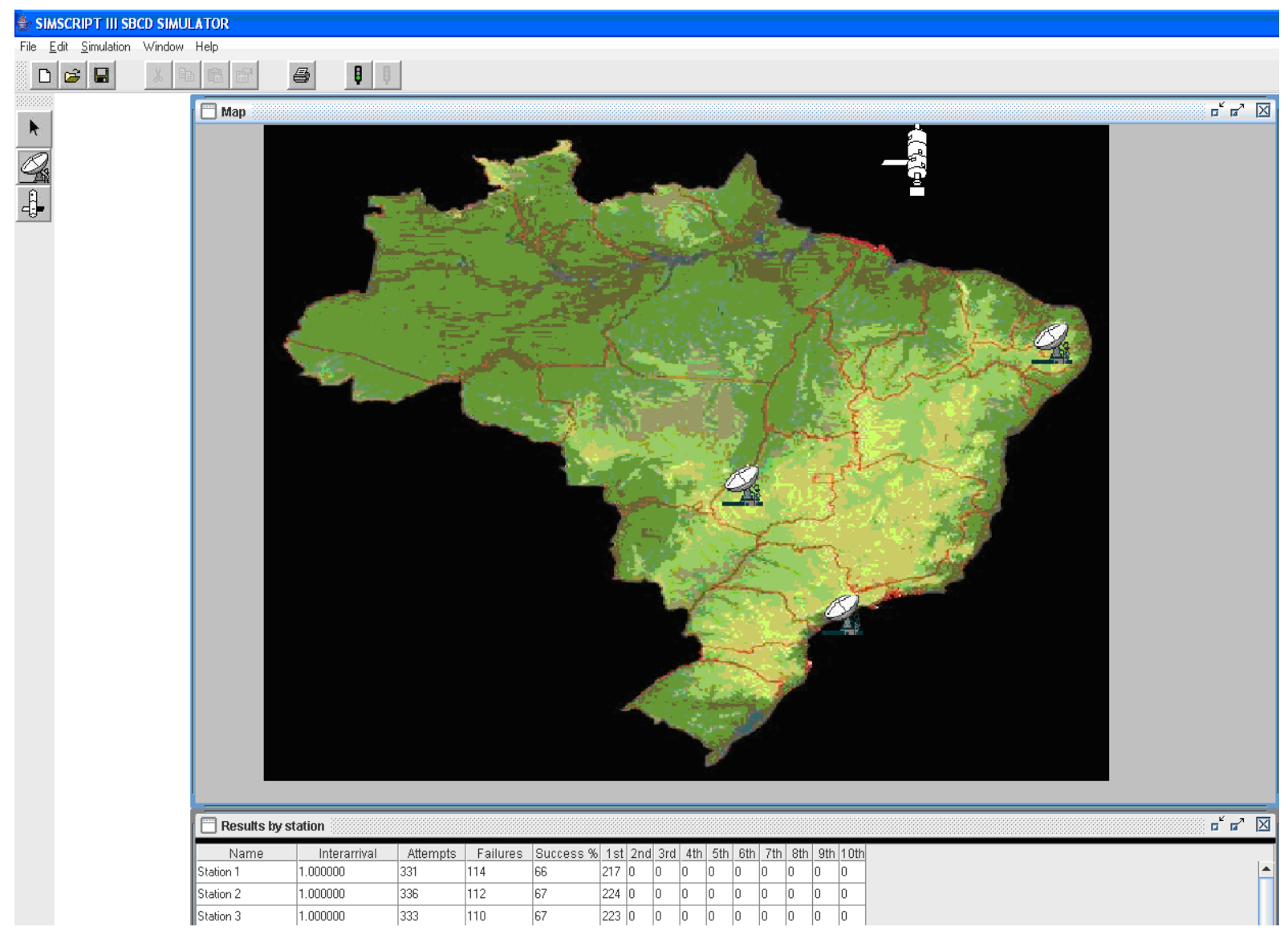

Figure 3: GUI of the SBCD Simuladors Prototype in SIMSCRIPT III 